\title{
Onset of quantum chaos in molecular systems and the zeros of the Husimi function
}

\author{
F. J. Arranz, ${ }^{1}$ L. Seidel, ${ }^{1}$ C. G. Giralda, ${ }^{1}$ R. M. Benito, ${ }^{1, *}$ and F. Borondo ${ }^{2, \dagger}$ \\ ${ }^{1}$ Grupo de Sistemas Complejos, Universidad Politécnica de Madrid, 28040 Madrid, Spain \\ ${ }^{2}$ Departamento de Química and Instituto de Ciencias Matemáticas (ICMAT), Universidad Autónoma de Madrid, 28049 Madrid, Spain
}

(Received 12 April 2013; published 3 June 2013)

\begin{abstract}
The distribution of zeros of the Husimi function has proven a useful tool in the characterization of regular and chaotic quantum states of dynamical systems. In a previous paper [Phys. Rev. E 82, 026201 (2010)], we showed how the quantum transition from order to chaos in three different molecular systems ( $\mathrm{LiCN}, \mathrm{HCN}$, and $\mathrm{HO}_{2}$ ) can be understood by means of the correlation diagram of eigenenergies versus the Planck's constant. An order-chaos frontier of scars (eigenstates localized over unstable and stable complementary, in the sense of the Poincaré-Birkhoff theorem, periodic orbits) was observed. In this paper, we show how the distribution of zeros of the Husimi function can be related to the onset of chaos in these molecular systems, showing a very interesting feature at the frontier of scars; namely, some zeros of the Husimi function localize over the stable and unstable fixed points corresponding to the two complementary periodic orbits, this representing the quantum equivalent to the Poincaré-Birkhoff theorem.
\end{abstract}

DOI: 10.1103/PhysRevE.87.062901

PACS number(s): 05.45.Mt, 05.45.Ac, 03.65.Sq

\section{INTRODUCTION}

In a seminal paper, Lebœuf and Voros [1] showed that the distribution of zeros of the Husimi function is a meaningful indicator of the regular or chaotic character of a state in quantum maps. Specifically, the distribution of zeros is onedimensional, with the zeros distributed along a line, for regular states, while it is two-dimensional, with the zeros spreading uniformly in Husimi phase space, for chaotic states.

Thereafter, some authors have extended these studies in quantum maps [2-4], and also these properties have been shown to exist in more realistic systems. The regular or chaotic behavior of quantum states has been characterized through the distributions of the Husimi zeros in the spin-boson interaction model $[5,6]$, in quantum billiards $[7,8]$, in one-dimensional autonomous (then integrable) systems $[9,10]$, in spin SternGerlach apparatus [11] (where it is possible to experimentally measure the zeros), in acoustic waveguides [12], and also in molecular systems [13-17].

In a previous paper [18], we have shown how the quantum order-chaos transition for three different molecular systems ( $\mathrm{LiCN}, \mathrm{HCN}$, and $\mathrm{HO}_{2}$ ) is seen in the correlation diagram of eigenenergies versus Planck's constant, where an order-chaos frontier of scars (eigenstates localized over unstable and stable complementary, in the sense of the Poincaré-Birkhoff theorem [19], periodic orbits) has been observed. In this study, we extend our previous results for the $\mathrm{LiCN}$ molecular system $[13,15,16]$ to the HCN (whose dynamics is slightly different) and $\mathrm{HO}_{2}$ (with behavior very different from LiCN) molecular systems, showing how the distribution of zeros of the Husimi function marks the onset of chaos in all these systems. As will be seen, there is a very interesting characteristic of the Husimi zeros at the frontier of scars, namely, some zeros localize over the stable and unstable fixed points corresponding to the two complementary periodic orbits, this representing

\footnotetext{
*gi.gsc@upm.es

†f.borondo@uam.es
}

a quantum correspondence with the Poincaré-Birkhoff theorem [4].

The organization of the paper is as follows. In the next section, we describe the three molecular systems under study, as well as the mathematical methods used in our calculations. In Sec. III, we present and discuss the most relevant results concerning the classical dynamics and the distribution of the Husimi zeros for our three molecular systems. Finally, we summarize our main conclusions in Sec. IV.

\section{MOLECULAR SYSTEMS DESCRIPTION AND CALCULATIONS}

\section{A. Molecular systems}

The systems studied in this work are triatomic molecules $A-B C$, where the length between $B$ and $C$ atoms can be fixed at its equilibrium value, due to the fact that the $B-C$ interaction is much stronger than the interactions with atom $A$, and then an adiabatic decoupling of the corresponding degree of freedom is possible. Namely, we study the motion of atom $A$ around the dimer $B C$. The corresponding Hamiltonian function for the purely vibrational, i.e., without rotation, dynamics is given in Jacobi coordinates by

$$
H=\frac{P_{R}^{2}}{2 \mu_{1}}+\frac{P_{\theta}^{2}}{2}\left(\frac{1}{\mu_{1} R^{2}}+\frac{1}{\mu_{2} r_{\mathrm{eq}}^{2}}\right)+V(R, \theta),
$$

where $\mu_{1}=m_{A}\left(m_{B}+m_{C}\right) /\left(m_{A}+m_{B}+m_{C}\right)$ and $\mu_{2}=$ $m_{B} m_{C} /\left(m_{B}+m_{C}\right)$ are reduced masses, $m_{X}$ are the corresponding atomic masses, $r_{\mathrm{eq}}$ is the fixed $B-C$ equilibrium length, $R$ is the length between the $B-C$ center of mass and atom $A$, and $\theta$ is the angle formed by the corresponding $R$ and $r_{\mathrm{eq}}$ directions, with $\theta=0$ and $\theta=180^{\circ}$ for the linear configurations $A-B C$ and $B C$ - $A$, respectively. $P_{R}$ and $P_{\theta}$ are the conjugate momenta, and $V(R, \theta)$ is the potential energy surface (PES) corresponding to the different molecular systems. It should be noted that the Hamiltonian function in Eq. (1) corresponds to a vibrational model for molecular systems where the interactions with the rotational and electronic degrees of freedom are neglected. 

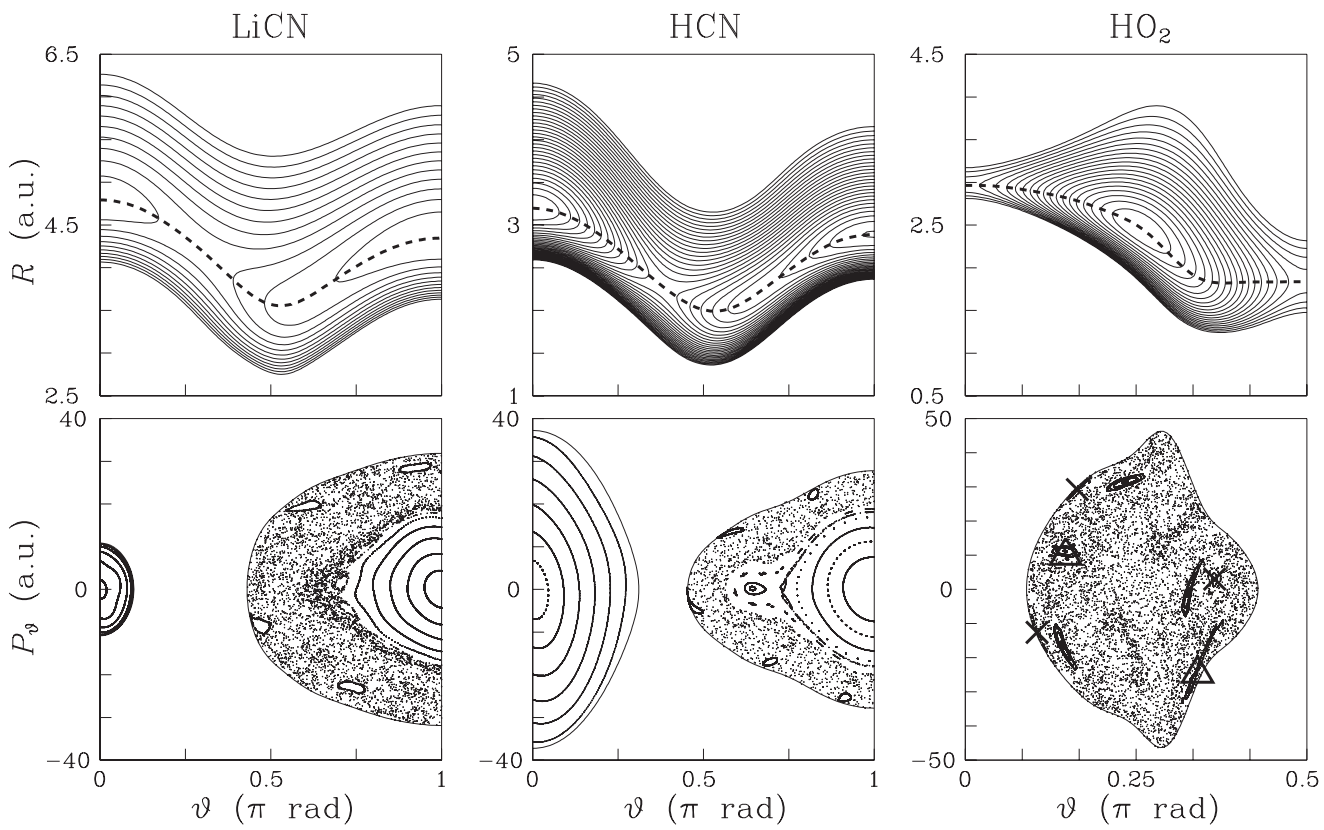

FIG. 1. (Top) Potential energy surfaces represented as contour plots spaced $1000 \mathrm{~cm}^{-1}$ for LiCN (left), $\mathrm{HCN}$ (middle), and $\mathrm{HO}_{2}$ (right) molecular systems. Potential energy zeros have been set at the absolute minimum of each surface. Also, the minimum energy paths have been plotted in dashed line. (Bottom) Composite Poincaré surfaces of section along the corresponding minimum energy path at energies of $2500 \mathrm{~cm}^{-1}$ for $\mathrm{LiCN}$ (left), $11200 \mathrm{~cm}^{-1}$ for $\mathrm{HCN}$ (middle), and $12500 \mathrm{~cm}^{-1}$ for $\mathrm{HO}_{2}$ (right). Notice the chains of islands near to energy boundaries corresponding to resonances, accounting symmetry, 1:8 for $\mathrm{LiCN}, 1: 10$ for $\mathrm{HCN}$, and 1:2 and 1:3 for $\mathrm{HO}_{2}$ (marked with triangles $(\triangle)$ and crosses $(\times)$, respectively).

In particular, we have studied the $\mathrm{Li}-\mathrm{CN}$ and $\mathrm{H}-\mathrm{CN}$ isomerizing systems, and the $\mathrm{H}-\mathrm{O}_{2}$ radical, all of them with highly nonlinear behavior at low energies but with different dynamical characteristics. The corresponding PES's, which have been taken from literature [20-22], are shown in Fig. 1 (top), where their topographic differences can be observed.

Notice that the PES for the LiCN molecule has two minima at $\theta=0$ and $\theta=180^{\circ}$, corresponding to the two linear isomers $\mathrm{Li}-\mathrm{CN}$ and $\mathrm{CN}-\mathrm{Li}$, and a saddle point at $\theta=52.6^{\circ}$, corresponding to the transition state, all of them connected by the minimum energy path (MEP). The most stable well is at $\theta=180^{\circ}$ and, as we will see in Sec. III A, this is the well where chaos starts.

The HCN PES is similar to that of $\mathrm{LiCN}$, but in this case the well at $\theta=0$, corresponding to the $\mathrm{H}-\mathrm{CN}$ linear isomer, is the most stable one. However, as in the $\mathrm{LiCN}$ case, chaos starts in the well at $\theta=180^{\circ}$. In this case, the saddle point is located at $\theta=68.4^{\circ}$.

Finally, the $\mathrm{HO}_{2}$ PES presents a very different structure. Notice that this system, due to the homonuclear character of the dimer O-O, has an additional symmetry plane (aside from the trivial one along the interatomic axis direction $r_{\mathrm{eq}}$, like in the LiCN and HCN systems) perpendicular to the interatomic axis. The PES has two symmetric minima at $\theta=46.2^{\circ}$ and $\theta=133.8^{\circ}$, and three saddle points located at $\theta=0,90^{\circ}$, and $180^{\circ}$, respectively. Note that the topography of $\mathrm{HO}_{2}$ PES implies quantum degeneracy, at least for low energies, where the two wells are disconnected and tunneling is negligible, the degenerate eigenstates being either symmetric or antisymmetric in respect to the additional symmetry plane.

\section{B. Classical calculations}

The Hamilton equations of motion corresponding to Eq. (1) are built and standard numerical integration is used to obtain trajectories for each molecular system. In order to get a suitable graphical representation of the phase space, we have calculated composite Poincaré surfaces of section (PSS's) along the MEP for different energies.

For this purpose, the coordinates $\left(R_{i}, \theta_{i}\right)$ of the MEP have been numerically calculated and then fitted to a Fourier series (to take advantage of the angular periodicity of the system), obtaining an analytical expression $R_{\mathrm{eq}}(\theta)=\sum_{k} c_{k} \cos k \theta$ (with $k$ even for $\mathrm{HO}_{2}$, due to the additional symmetry) suitable for differential transformations. Then, the canonical transformation

$$
\begin{aligned}
\rho & =R-R_{\mathrm{eq}}(\theta), \quad \vartheta=\theta, \\
P_{\rho} & =P_{R}, \quad P_{\vartheta}=P_{\theta}+P_{R}\left[d R_{\mathrm{eq}}(\theta) / d \theta\right],
\end{aligned}
$$

is made, so that, for a given energy $E$, a PSS along the MEP is defined in the $\left(\vartheta, P_{\vartheta}\right)$ coordinates, by making $\rho=0$ and choosing an arbitrary branch (the negative one in our calculations) in the second-order equation for $P_{\rho}$ that arise from the Hamiltonian conservation $H\left(\rho, \vartheta, P_{\rho}, P_{\vartheta}\right)=E$. Finally, all the PSS points are folded into the interval $\vartheta \in(0, \pi)$ to take into account the symmetry of the molecular systems.

\section{Quantum calculations}

In order to obtain the eigenfunctions and eigenenergies of the Hamiltonian operator corresponding to Eq. (1), the discrete variable representation-distributed Gaussian basis 
method of Bačić and Light [23] has been used. In this way, the approximately 900 low-lying eigenfunctions $\langle R \theta \mid n\rangle$, $n=1, \ldots, 900$, with its eigenenergies converged to within $0.1 \mathrm{~cm}^{-1}$, have been obtained.

Moreover, and as it is well known (see, e.g., Ref. [24]), for a state $|\psi\rangle$ from a $N$ degrees of freedom system, the Husimi function $\mathcal{H}(\mathbf{z})$ can be expressed as the probability density in the coherent state representation, that is

$$
\mathcal{H}(\mathbf{z})=\pi^{-N}|\langle\mathbf{z} \mid \psi\rangle|^{2} .
$$

The coherent states $|\mathbf{z}\rangle=\left|z_{1}\right\rangle \otimes \cdots \otimes\left|z_{N}\right\rangle$ are defined as the eigenstates of the annihilation operator $\widehat{a}_{k}=2^{-1 / 2}\left[\alpha_{k} \widehat{X}_{k}+\right.$ $\left.i \widehat{P}_{k} /\left(\alpha_{k} \hbar\right)\right]$, whose eigenvalues are $z_{k}=2^{-1 / 2}\left[\alpha_{k} \bar{x}_{k}+\right.$ $\left.i \bar{p}_{k} /\left(\alpha_{k} \hbar\right)\right]$. The parameters $\alpha_{k}$ are given by $\alpha_{k}=\sqrt{m_{k} \omega_{k} / \hbar}$, as a function of the mass $m_{k}$ and the harmonic frequency $\omega_{k}$ of the $k$ th degree of freedom, and the Planck's constant $\hbar$. Notice that the mean position and the mean momentum appear, respectively, in the real and imaginary part of the eigenvalues, that is, $\bar{x}_{k} \equiv\left\langle z_{k}\left|\widehat{X}_{k}\right| z_{k}\right\rangle$ and $\bar{p}_{k} \equiv\left\langle z_{k}\left|\widehat{P}_{k}\right| z_{k}\right\rangle$. Therefore, the coherent state representation $\langle\mathbf{z} \mid \psi\rangle$ can be calculated from the position representation $\langle\mathbf{x} \mid \psi\rangle$ by means of a basis set change

$$
\langle\mathbf{z} \mid \psi\rangle=\int d^{N} x\langle\mathbf{z} \mid \mathbf{x}\rangle\langle\mathbf{x} \mid \psi\rangle,
$$

where $\langle\mathbf{z} \mid \mathbf{x}\rangle=\left\langle z_{1} \mid x_{1}\right\rangle \cdots\left\langle z_{N} \mid x_{N}\right\rangle$ is the harmonic oscillator coherent packet given by

$$
\left\langle x_{k} \mid z_{k}\right\rangle=\left(\frac{\alpha_{k}}{\sqrt{\pi}}\right)^{1 / 2} e^{-\frac{1}{2}\left(\left|z_{k}\right|^{2}-z_{k}^{2}\right)} e^{-\frac{1}{2}\left(\alpha_{k} x_{k}-\sqrt{2} z_{k}\right)^{2}} .
$$

Accordingly, we have obtained the coherent state representation of the eigenstates of our molecular systems $\langle\mathbf{z} \mid n\rangle$ by using the basis set change in Eq. (4), but expressed in Jacobi instead of Cartesian coordinates, and then we have built the Husimi function $\mathcal{H}\left(R, \theta, P_{R}, P_{\theta}\right)$ through Eq. (3), taking the coordinates and momenta of real and imaginary parts instead of using the complex variables. Notice that, in this case, the normalization factor $\pi^{-N}$ in Eq. (3) should be replaced by $(2 \pi \hbar)^{-N}$.

In order to get a suitable graphical representation, and to be able to compare with the classical calculations, a quantum Poincaré surface of section (QPSS), $\mathcal{H}_{\mathrm{s}}\left(\vartheta, P_{\vartheta}\right)$, has been calculated by making, similarly to the classical PSS, the canonical transformation given in Eq. (2) and setting $\rho=0$ and $P_{\rho}$ chosen as in the classical case (negative branch).

Last, we have calculated the zeros of the QPSS $\mathcal{H}_{\mathrm{s}}\left(\vartheta, P_{\vartheta}\right)$ by using the properties of the index of a curve [25]. Let $\mathcal{T}: \mathbf{x} \mapsto \mathbf{y}$ be a continuous transformation $\mathbb{R}^{2} \stackrel{\mathcal{T}}{\rightarrow} \mathbb{R}^{2}$, then the index of a closed curve defined in the origin space $\mathbf{x}$ equals the number of zeros in the image space $\mathbf{y}$ enclosed by that curve. Moreover, the index of a curve is the winding number of the transformed curve around the coordinate origin of the image space. Thus, taking into account that the Husimi QPSS, same as the Husimi function [see Eq. (3)], is the squared modulus of a complex number

$$
\mathcal{H}_{\mathrm{s}}\left(\vartheta, P_{\vartheta}\right)=(2 \pi \hbar)^{-2}\left[\operatorname{Re}^{2}(\phi)+\operatorname{Im}^{2}(\phi)\right],
$$

we have considered the transformation

$$
\mathcal{T}:\left(\vartheta, P_{\vartheta}\right) \mapsto(\operatorname{Re}(\phi), \operatorname{Im}(\phi))
$$

Then, the origin space $\left(\vartheta, P_{\vartheta}\right)$ has been divided into square cells, and the number of zeros contained in each cell numerically calculated by counting the winding number of the transformed cell in the image space $(\operatorname{Re}(\phi), \operatorname{Im}(\phi))$. More details of this technique of calculation of zeros are given in Ref. [14].

\section{RESULTS AND DISCUSSION}

\section{A. Classical dynamics}

Representative composite PSS's for each molecule at intermediate energies below the saddles are shown at the bottom part of Fig. 1. It can be seen that these molecular systems have a mixed phase space; that is, in all three systems we can observe regular regions corresponding to tori and chains of islands, and also chaotic regions. Notice that $\mathrm{HO}_{2}$ shows the most chaotic dynamics.

The LiCN system exhibits a regular dynamics around the fixed points corresponding to the stable periodic orbits (PO's) contained in the symmetry planes at $\vartheta=0,180^{\circ}$, and associated to the two isomer potential wells. Also, we can observe that the dynamics is very regular in the potential well at $\vartheta=0$, and present a mixed character in the well around $\vartheta=180^{\circ}$, which is the most stable one. Special attention deserves the chain of eight islands (only four of them are visible in the $0<\vartheta<180^{\circ}$ range of the figure) near the energy boundary, corresponding to a 1:8 stable PO, together with the chain of eight hyperbolic fixed points interspersed in the chain of islands, not visible in the figure, corresponding to a 1:8 unstable PO. As we will show in Sec. III B3, a very interesting feature of the zeros of the Husimi function is related to these two complementary PO's.

On the other hand, the $\mathrm{HCN}$ system exhibits a behavior similar to LiCN. It also shows a very regular dynamics in the potential well at $\vartheta=0$ and a mixed structure in the well at $\vartheta=180^{\circ}$, which in this case is the less stable one. That is, in the HCN system the most stable well is also the well with more regular dynamics. Observe that in this system, as in the LiCN case, there exists a chain of islands near the energy boundary, but in this case the chain has ten islands (only five of them are shown in the range of the figure), corresponding to a 1:10 stable $\mathrm{PO}$, and there exists also the corresponding 1:10 unstable PO. As for the LiCN system, these two complementary PO's are closely related with the zeros of the Husimi function of scarred eigenstates.

Moreover, the $\mathrm{HO}_{2}$ system shows a very different dynamical behavior. As it is described in Ref. [18], the principal elliptic fixed point bifurcates at very low energy becoming hyperbolic, and only some chains of islands remain as stable structures. Anyway, the chains of islands near the energy boundary marked in the figure play a similar role, regarding the zeros of the Husimi function, to that of the corresponding chains of islands in the $\mathrm{LiCN}$ and $\mathrm{HCN}$ systems. It should be remarked here that even though these two chains of islands correspond to 1:2 and 1:3 stable PO's, both appear at the same saddle node bifurcation as unstable and stable PO's, respectively, but as energy increases the 1:2 PO becomes stable. 

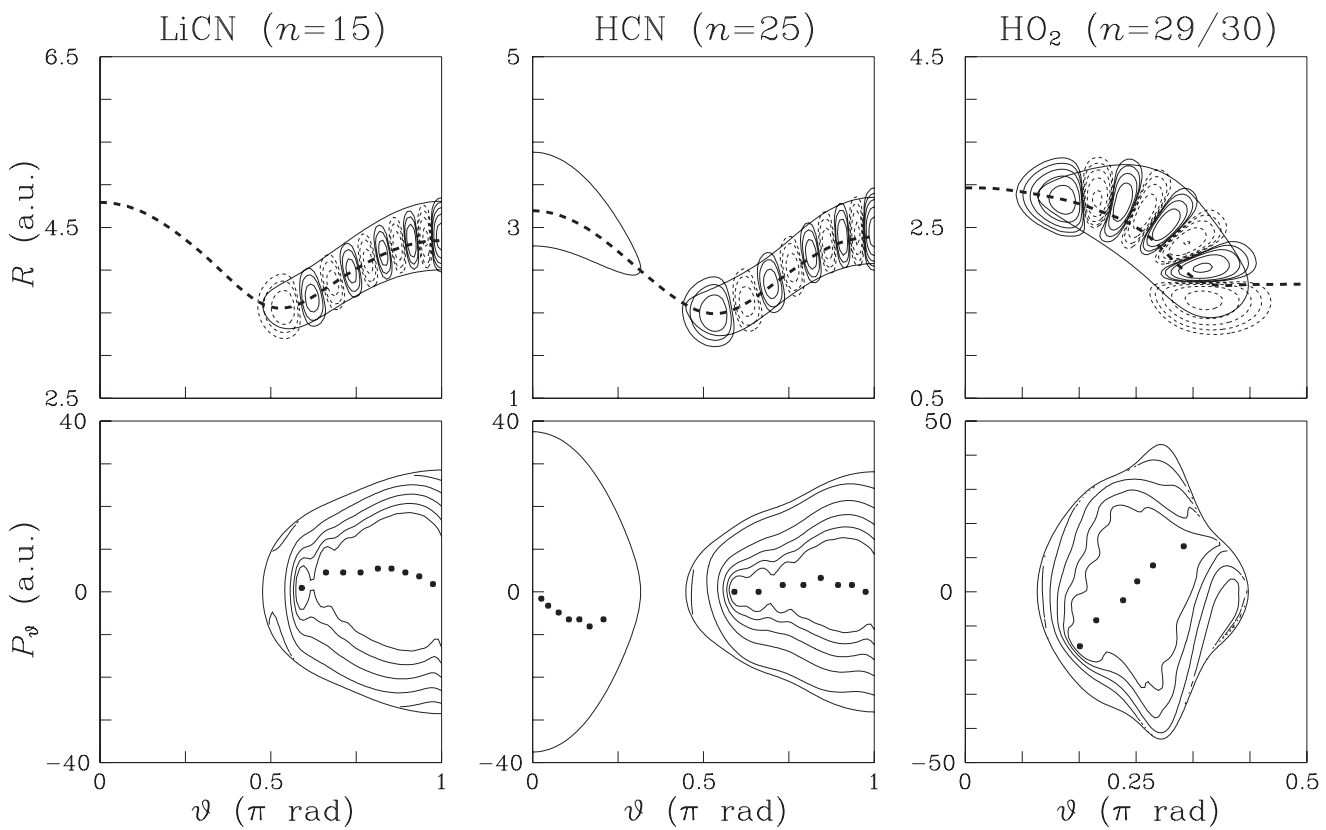

FIG. 2. Regular eigenstates. (Top) Wavefunctions in position representation depicted as contour plots for eigenstates $n=15$ of LiCN (left), $n=25$ of $\mathrm{HCN}$ (middle), and $n=29 / 30$ of $\mathrm{HO}_{2}$ (right) molecular systems. The corresponding eigenenergy contours and minimum energy paths have also been plotted in continuous and dashed line, respectively. (Bottom) Quantum Poincaré surfaces of section along the corresponding minimum energy path of the Husimi functions represented as contour plots for the same eigenstates shown in the top panels. The position of the zeros has been marked with dots $(\cdot)$.

\section{B. Quantum eigenstates}

As will be shown below, the distribution of zeros of the Husimi function characterizes the regular or chaotic behavior of the eigenstates in a similar way as the nodal pattern does for the wavefunction (in position or momentum representation) $[26,27]$. Indeed, the zeros of the Husimi function are the nodal pattern of the squared modulus of the wavefunction in the coherent state representation. However, the zeros of the Husimi function have been shown to be a particularly sensitive indicator, capable of detecting characteristics that are not detectable by the nodal pattern of the wavefunction $[13,16]$. In addition, the zeros corresponding to the eigenstates at the order-chaos frontier of scars [18] have an interesting feature related to the fixed points of the involved PO's.

\section{Regular eigenstates}

Three typical regular eigenstates, one for each molecule, are presented in Fig. 2, where the wavefunctions in position representation and the Husimi QPSS's with its corresponding zeros are shown. In all cases, the eigenstate number starts at $n=1$ for the ground state.

For the LiCN system we observe a regular wavefunction excited along the MEP, that is, with its nodal lines perpendicular to the MEP, corresponding to the eigenstate $n=15$. Note that the number of these nodal lines (into the $0<\theta<180^{\circ}$ range of the figure) is $n_{\perp}=9$. Since there is not transversal excitation, the number of nodal lines parallel to the MEP is $n_{\|}=0$. Let us now examine the Husimi QPSS and its distribution of zeros. We see that the highest probability density is localized along the energy boundary, the maximum (and minimum) momentum $P_{\vartheta}$ being the most probable for each $\vartheta$ value, as corresponds to a state without transversal excitation. Then, this is an eigenstate quantized over the most external torus of the PSS. Also, the distribution of zeros is one-dimensional, namely, the zeros are located along a line, as expected for a regular state. Notice that the values of the $\vartheta$ coordinates at the zeros approximately match the $\theta$ coordinates of the wavefunction nodal lines at the MEP [remember that $\vartheta=\theta$ in the canonical transformation of Eq. (2)], so that the number of wavefunction nodal lines $n_{\perp}$ and the number of Husimi function zeros $n_{z}$ is the same, $n_{\perp}=n_{z}=9$. Furthermore, the $P_{\vartheta}$ coordinates of the zeros are approximately null, $P_{\vartheta} \approx 0$, this also being characteristic of regular states.

In the HCN system, features similar to the $\mathrm{LiCN}$ system are observed. In this case, the regular wavefunction excited along the MEP, corresponding to the eigenstate $n=25$, has the quantum numbers $\left(n_{\|}, n_{\perp}\right)=(0,8)$, the highest probability density of the Husimi QPSS is also localized along the energy boundary, the eigenstate being quantized over the most external torus, and the distribution of zeros is onedimensional, with $n_{\perp}=n_{z}=8$, and the $\vartheta$ coordinates of the zeros approximately matches the $\theta$ coordinates of the wavefunction nodal lines at the MEP, the $P_{\vartheta}$ coordinates of the zeros being approximately null. Note that, due to the fact that the amplitude of the wavefunction in the well at $\theta=0$ is negligible, there are not visible contour lines of the wavefunction nor the Husimi QPSS shown in the figure, but the nodal pattern is visible in the zeros of the Husimi QPSS.

The $\mathrm{HO}_{2}$ system is, however, very different from the previous cases. First, the wavefunction and Husimi QPSS shown in Fig. 2 correspond to the two degenerate eigenstates $n=29$ and $n=30$, symmetric and antisymmetric, respectively, to its symmetry plane at $\theta=90^{\circ}$. Besides, in 

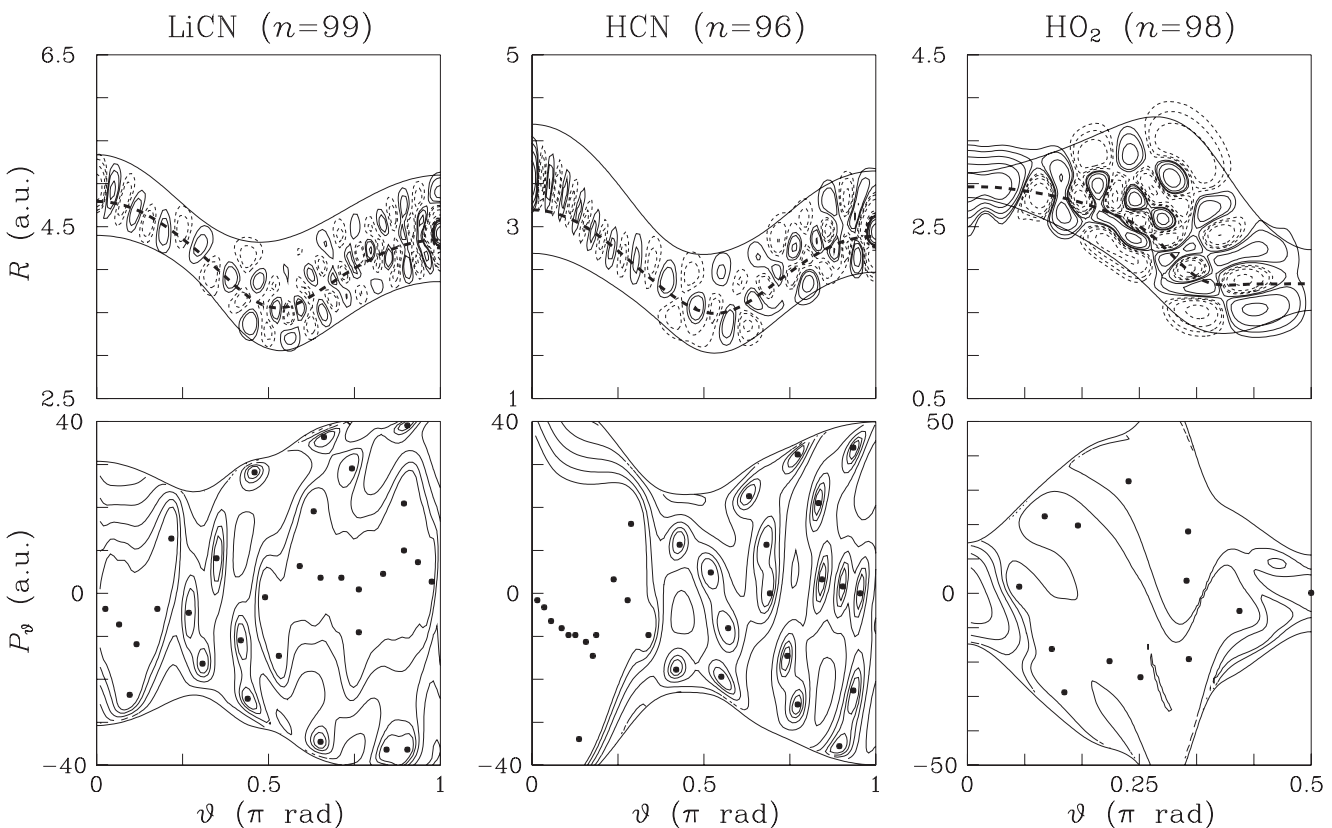

FIG. 3. Chaotic eigenstates. Same as described in the legend of Fig. 2 for eigenstates $n=99$ of LiCN (left), $n=96$ of HCN (middle), and $n=98$ of $\mathrm{HO}_{2}$ (right) molecular systems.

this system there are not purely regular eigenstates, except perhaps the ground and first excited states. As discussed in Sec. III A, the principal elliptic fixed point bifurcates at very low energy (approximately the eigenenergy of the first excited eigenstate), so that only the ground and the first excited eigenstates are quantized over the principal tori, the subsequent eigenstates being quantized over the surviving chains of islands. Therefore, we can observe that the wavefunction of eigenstate $n=29 / 30$ is not exactly excited along the MEP, the last node being parallel, rather than perpendicular, to the MEP. Furthermore, the Husimi QPSS has a maximum clearly located around $\left(\vartheta, P_{\vartheta}\right)=\left(70^{\circ},-5\right.$ a.u. $)$, instead of having its maxima localized along the energy boundary. However, this state can be considered mostly regular since the distribution of zeros is one-dimensional, although with $n_{\perp}=7 \neq n_{z}=6$. Note that this inequality is due to the parallel position of the last node with respect to the MEP. Also observe that, unlike for the purely regular eigenstates of $\mathrm{LiCN}$ and $\mathrm{HCN}$, the momentum coordinates of the zeros are not approximately null in this case.

\section{Chaotic eigenstates}

In Fig. 3 we show three examples of eigenstates from LiCN, $\mathrm{HCN}$, and $\mathrm{HO}_{2}$ systems that can be considered as irregular or chaotic states.

For the LiCN and HCN systems, the eigenstates $n=99$ and $n=96$, respectively, have been represented. We can observe that, in both cases, the corresponding wavefunctions (upper panels) have an irregular nodal pattern in the region around the well $\theta=180^{\circ}$, while in the region around the well $\theta=0$ they are roughly regular, in agreement with the classical behavior described in Sec. III A, and also shown in the PSS's of Fig. 1 (bottom). Moreover, the Husimi QPSS's of both systems show a delocalized distribution of its maxima, and a two-dimensional widespread distribution of the zeros, into the well at $\theta=180^{\circ}$, as expected for a chaotic state, and more localized maxima and a near to one-dimensional distribution of zeros into the well at $\theta=0$.

On the other hand, the represented eigenstate $n=98$ of the $\mathrm{HO}_{2}$ system shows a completely irregular nodal pattern in its wavefunction, and a delocalized distribution of the maxima in its Husimi QPSS, with the zeros spreading out in a twodimensional distribution. Notice that for this eigenstate there is no longer energy degeneracy.

\section{Scarred eigenstates}

Next, we discuss the behavior of the $\mathrm{LiCN}, \mathrm{HCN}$, and $\mathrm{HO}_{2}$ systems at the frontier of scars between order and chaos, as was defined in Ref. [18]. We will focus our attention on the corresponding distribution of zeros of the Husimi QPSS.

As energy increases, the first nonregular eigenstates of the LiCN system are the states $n=24$ and $n=25$, shown in Fig. 4 . We can see how the corresponding wavefunctions are excited along the 1:8 PO's (superimposed in the figure) rather than along the MEP, as it would be the case for regular states, with $n_{\perp}=12$ nodal lines perpendicular to the corresponding PO in both states. Notice that the PO's in eigenstates $n=24$ and $n=25$ are, respectively, unstable and stable complementary (in the sense of the Poincaré-Birkhoff theorem [19]) PO's. Namely, the eigenstate $n=24$ is a scar, and the eigenstate $n=25$ is a localized state. ${ }^{1}$ Particularly interesting are the Husimi QPSS's of these states. As we can see, the maxima of the Husimi QPSS's are located around the fixed points of the corresponding PO's, that is, these states are not localized over the PO's in the configuration space only, but they are also

\footnotetext{
${ }^{1}$ As originally defined by Heller [28], the term scar coined by him applies to states localized over unstable PO's.
} 


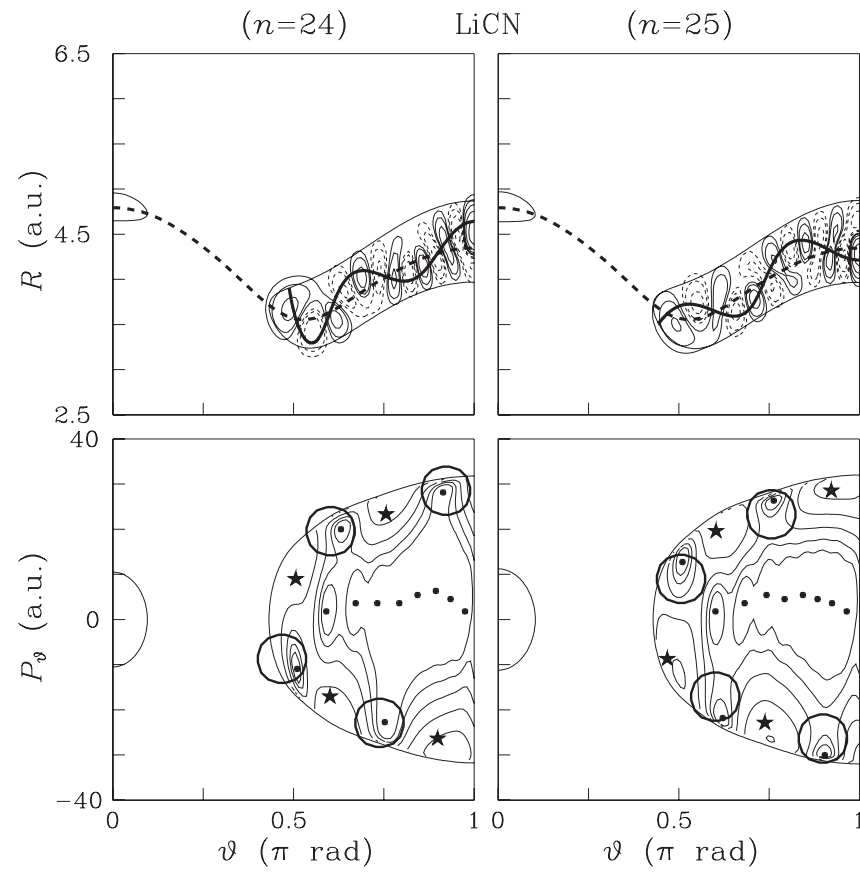

FIG. 4. Scarred eigenstates. (Top) Wavefunctions in position representation depicted as contour plots for eigenstates $n=24$ (left) and $n=25$ (right) of LiCN molecular system. The corresponding eigenenergy contours and minimum energy paths have been plotted in continuous and dashed line, respectively. Also, the two complementary scarred periodic orbits have been plotted in thick line. (Bottom) Quantum Poincaré surfaces of section along the corresponding minimum energy path of the Husimi functions represented as contour plots for eigenstates $n=24$ (left) and $n=25$ (right) of LiCN. Zeros have been plotted as dots (•). Also, the fixed points of the corresponding scarred periodic orbit and its complementary one have been plotted as stars $(\star)$ and big circles $(O)$, respectively.

localized in the phase space. Furthermore, the number of zeros equals the number of wavefunction nodal lines, $n_{z}=n_{\perp}=12$, and the zeros have a one-dimensional distribution with $P_{\vartheta} \approx 0$, as for a regular state, except 4 of them (as many zeros as PO's fixed points) that are localized around the fixed points of the complementary PO. Note that these complementary PO's correspond to those discussed in Sec. III A and indicated in the caption of Fig. 1.

For the HCN system, similar results to those for the $\mathrm{LiCN}$ system are found. In Fig. 5, the eigenstates $n=44$ and $n=45$ corresponding to the frontier of scars are shown. We can see that they have two clearly differentiated parts: one into the well at $\theta=0$ and the other into the well around $\theta=180^{\circ}$. The part around $\theta=0$ is mostly regular (and similar) for both states, corresponding to the excitation along the MEP, namely, with the nodal lines of the wavefunction being perpendicular to the MEP, and with a mainly one-dimensional distribution of the zeros of the Husimi QPSS. Notice that the number of wavefunction nodal lines equals the number of Husimi QPSS zeros, as expected. Moreover, the part around $\theta=180^{\circ}$ is where scarring takes place. Indeed, in this part the eigenstates $n=44$ and $n=45$ are excited along the complementary (unstable and stable, respectively) 1:10 PO's, with $n_{\perp}=12$ wavefunctions nodal lines perpendicular to the corresponding PO in both states. We can observe that the localization over the

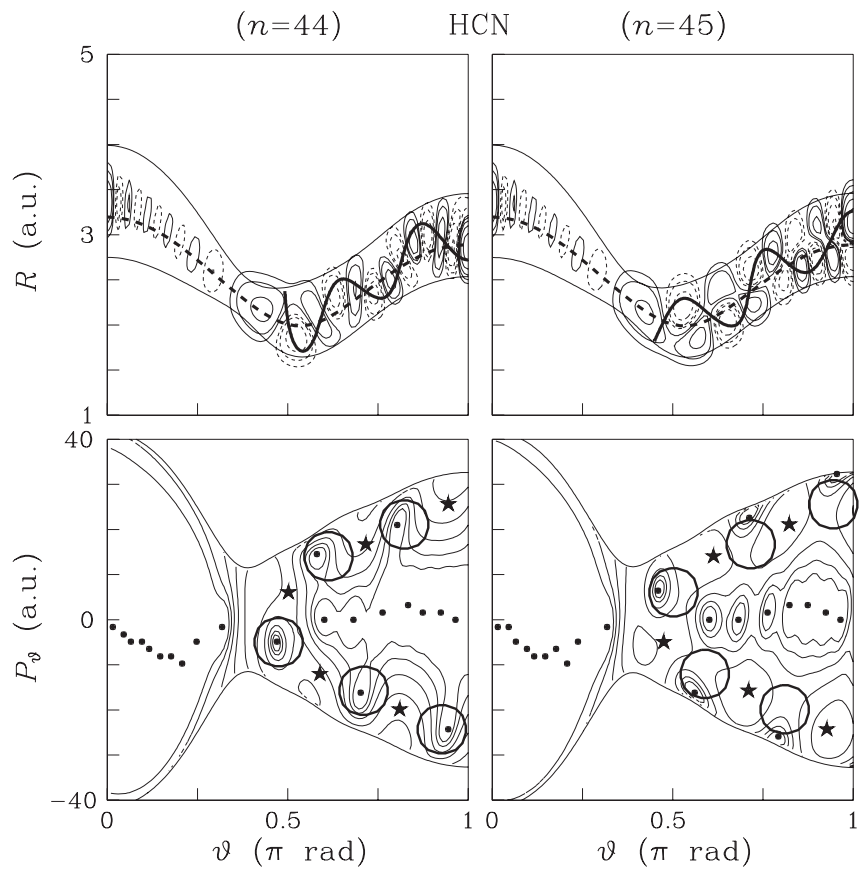

FIG. 5. Scarred eigenstates. Same as described in the legend of Fig. 4 for eigenstates $n=44$ (left) and $n=45$ (right) of HCN molecular system.

stable PO is not as clear in the wavefunction of the state $n=45$, as in the corresponding Husimi QPSS representation, where its 5 maxima are seen to approximately coincide with the PO fixed points. The interesting behavior of the Husimi QPSS zeros for scarred states previously shown in LiCN system also takes place here, namely, the number of zeros equals the number of wavefunction nodal lines, $n_{z}=n_{\perp}=12$, and its distribution is one-dimensional, except for 5 of them, which are localized around the 5 fixed points of the complementary PO.

On the other hand, the $\mathrm{HO}_{2}$ system, although being very different to $\mathrm{LiCN}$ and $\mathrm{HCN}$, also shows the above behavior at the frontier of scars. For this system, we have represented the eigenstates $n=49 / 50$ and $n=53 / 54$ (degeneracy still exists at these eigenenergies) in Fig. 6, where it can be observed how the corresponding wavefunctions appear mostly excited along the 1:3 and 1:2 PO's, with $n_{\perp}=10$ excitations in both cases. As explained in Sec. III A, the stable and unstable PO's arise at a saddle node bifurcation, but thereafter the unstable one becomes stable. Then, strictly speaking, these eigenstates would not be scars, but localized states over stable PO's. However, the features of the Husimi QPSS shown above for scarred states of the $\mathrm{LiCN}$ and HCN systems are also seen in the $\mathrm{HO}_{2}$ system. We can observe that the maxima of the Husimi QPSS's are located around the fixed points of the corresponding PO's, namely, these states are localized over the PO's not only in configuration space but also in phase space. Besides, the zeros have a one-dimensional distribution, except 2 of them for the state $n=49 / 50$ that are localized around the 2 fixed points of the 1:2 PO, and 3 zeros of the state $n=53 / 54$ that are localized around the 3 fixed points of the 1:3 PO. Nevertheless, as in the regular eigenstates of this system, the number of zeros is not equal to the number of 


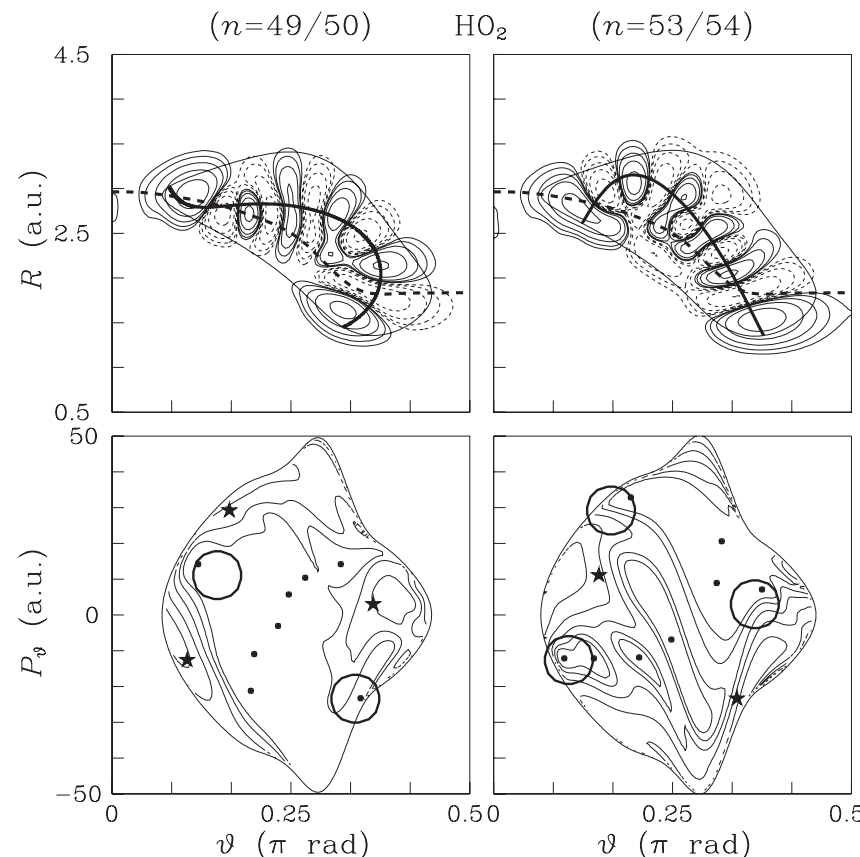

FIG. 6. Scarred eigenstates. Same as described in the legend of Fig. 4 for eigenstates $n=49 / 50$ (left) and $n=53 / 54$ (right) of $\mathrm{HO}_{2}$ molecular system.

wavefunction nodal lines, $n_{\perp}=10 \neq n_{z}=8$, due to reasons similar to those of the regular case.

\section{CONCLUDING REMARKS}

The distribution of zeros of the Husimi function has been shown to be a useful indicator of the onset of quantum chaos in quantized maps [1,3,4]. Particularly, the distribution is one-dimensional for regular states, and it is two-dimensional (spread in Husimi phase space) for chaotic states.

In this paper, we have extended our previous results about the zeros of the Husimi function in the molecular system LiCN $[13,15,16]$ to two different molecular systems: the slightly different system $\mathrm{HCN}$ and the very different system $\mathrm{HO}_{2}$. Classically, for a given excitation energy, all of them have a mixed type phase space, that is, a phase space composed of regular tori, chains of islands, and chaotic regions, the $\mathrm{HO}_{2}$ system being the most chaotic of all cases considered here.
Indeed, at intermediate energies, the $\mathrm{LiCN}$ and $\mathrm{HCN}$ systems maintain their principal elliptic fixed point, associated to the corresponding minimum of the PES, which is not the case for $\mathrm{HO}_{2}$, where its principal elliptic fixed point bifurcates at very low energy. Moreover, our study is carried out in a suitable quantum PSS of the Husimi function.

For the purely regular eigenstates of $\mathrm{LiCN}$ and $\mathrm{HCN}$ systems, i.e., those states quantized over tori around the principal elliptic fixed point, the distribution of zeros is one-dimensional, with momentum coordinates approximately null, and position coordinates corresponding to the nodal lines of the wavefunction in position representation. Thus, the number of zeros equals the number of wavefunction nodal lines. For the mostly regular eigenstates of the $\mathrm{HO}_{2}$ system, i.e., those states quantized over the surviving chains of islands, the distribution of zeros is also one-dimensional, but in this case the momentum coordinates are not approximately null. Besides, the position coordinates correspond to the nodal lines of the wavefunction in position representation, although here, due to the non purely regular character of the states, the number of zeros is not exactly the same as the number of wavefunction nodal lines. However, the distribution of zeros distinguishes between purely regular and mostly regular eigenstates.

On the other hand, for the chaotic eigenstates of the three systems, i.e., those states not quantized over tori, the distribution of zeros is two-dimensional, spreading out in the available Husimi phase space.

Finally, the most interesting result arises at the frontier of scars between order and chaos unveiled in our previous paper [18]. This frontier is formed by pairs of eigenstates that are quantized over two complementary (in the sense of the Poincaré-Birkhoff theorem [19]) stable and unstable periodic orbits. In this case, the distribution of zeros is onedimensional, like for regular states, except a number of zeros equal to the number of fixed points has the complementary periodic orbit that are localized around these fixed points, this representing the quantum equivalent to the Poincaré-Birkhoff theorem.

\section{ACKNOWLEDGMENT}

This research was supported by the Ministry of Economy and Competitiveness-Spain under Grant No. MTM201239101.
[1] P. Lebœuf and A. Voros, J. Phys. A 23, 1765 (1990).

[2] S. Nonnenmacher, Nonlinearity 10, 1569 (1997).

[3] S. Nonnenmacher and A. Voros, J. Stat. Phys. 92, 431 (1998).

[4] D. A. Wisniacki, M. Saraceno, F. J. Arranz, R. M. Benito, and F. Borondo, Phys. Rev. E 84, 026206 (2011).

[5] M. B. Cibils, Y. Cuche, P. Lebœuf, and W. F. Wreszinski, Phys. Rev. A 46, 4560 (1992).

[6] E. Romera, R. del Real, and M. Calixto, Phys. Rev. A 85, 053831 (2012).

[7] J. M. Tualle and A. Voros, Chaos Solitons Fractals 5, 1085 (1995).
[8] D. Biswas and S. Sinha, Phys. Rev. E 60, 408 (1999).

[9] H. J. Korsch, C. Müller, and H. Wiescher, J. Phys. A 30, L677 (1997).

[10] F. Toscano and A. M. O. de Almeida, J. Phys. A 32, 6321 (1999).

[11] J. P. Amiet and S. Weigert, J. Opt. B 1, L5 (1999).

[12] L. E. Kon'kov, D. V. Makarov, E. V. Sosedko, and M. Y. Uleysky, Phys. Rev. E 76, 056212 (2007).

[13] F. J. Arranz, F. Borondo, and R. M. Benito, Phys. Rev. E 54, 2458 (1996).

[14] F. J. Arranz, F. Borondo, and R. M. Benito, J. Mol. Structure (Theochem) 426, 87 (1998). 
[15] F. J. Arranz, F. Borondo, and R. M. Benito, Phys. Rev. Lett. 80 944 (1998).

[16] F. J. Arranz, R. M. Benito, and F. Borondo, J. Chem. Phys. 123, 044301 (2005).

[17] F. J. Arranz, Z. S. Safi, R. M. Benito, and F. Borondo, Eur. Phys. J. D 60, 279 (2010).

[18] F. J. Arranz, L. Seidel, C. G. Giralda, R. M. Benito, and F. Borondo, Phys. Rev. E 82, 026201 (2010).

[19] A. J. Lichtenberg and M. A. Lieberman, Regular and Chaotic Dynamics (Springer-Verlag, Berlin, 1992).

[20] R. Essers, J. Tennyson, and P. E. S. Wormer, Chem. Phys. Lett. 89, 223 (1982).
[21] J. N. Murrell, S. Carter, and L. O. Halonen, J. Mol. Spectrosc. 93, 307 (1982).

[22] A. J. C. Varandas, J. Brandão, and L. A. M. Quintales, J. Phys. Chem. 92, 3732 (1988).

[23] Z. Bačić and J. C. Light, J. Chem. Phys. 85, 4594 (1986).

[24] Hai-Woong Lee, Phys. Rep. 259, 147 (1995).

[25] V. I. Arnold, Ordinary Differential Equations (Springer-Verlag, Berlin, 1992).

[26] R. M. Stratt, N. C. Handy, and W. H. Miller, J. Chem. Phys. 71, 3311 (1979).

[27] N. de Leon, M. J. Davis, and E. J. Heller, J. Chem. Phys. 80, 794 (1984).

[28] E. J. Heller, Phys. Rev. Lett. 53, 1515 (1984). 